\title{
Shape-dependent magnetization reversal processes and flux-closure configurations of microstructured epitaxial $\mathrm{Fe}(110)$ elements
}

\author{
C. König, M. Sperlich, R. Heinesch, R. Calarco, J. O. Hauch, U. Rüdiger, ${ }^{\text {a) }}$ and \\ G. Güntherodt \\ II. Physikalisches Institut, RWTH Aachen, 52056 Aachen, Germany \\ S. Kirsch \\ Tieftemperaturphysik, Gerhard-Mercator-Universität Duisburg, 47048 Duisburg, Germany \\ B. Özyilmaz and A. D. Kent \\ Department of Physics, New York University, 4 Washington Place, New York, New York 10003
}

(Received 18 July 2001; accepted for publication 20 September 2001)

\begin{abstract}
The magnetization reversal processes and magnetic domain states of microstructured epitaxial $\mathrm{Fe}(110)$ elements have been investigated by magnetic-force microscopy and longitudinal Kerr hysteresis loop measurements. The characteristic micromagnetic behavior, such as coercive and nucleation fields, can be tailored by taking advantage of the pronounced uniaxial anisotropy and by varying the shape of the elements. The magnetic domain states of rectangular and diamond-shaped elements with lateral dimensions of $1.5 \mu \mathrm{m} \times 0.5 \mu \mathrm{m}$ have been investigated after magnetic saturation along the long and short axes of the elements. The observed flux-closure domain states have been compared with micromagnetic simulations.
\end{abstract}

With the recent development of magnetic random access memories (MRAMs) and magnetic-field sensors based on submicron magnetic elements, there is a great deal of interest in understanding and controlling the micromagnetic properties of such elements. ${ }^{1-3}$ The presence of stable and metastable magnetic domain configurations as a function of the magnetic history and the shape dependence of the magnetization reversal of defined magnetic elements have to be understood for a reproducible fabrication of magnetic storage devices on the basis of small magnetic elements.

As a model system for investigating the micromagnetic properties of submicron elements, 25-nm-thick epitaxial $\mathrm{Fe}(110)$ films were grown on $(11 \overline{2} 0)$ oriented $\mathrm{Al}_{2} \mathrm{O}_{3}$ substrates using molecular-beam-epitaxy techniques in ultrahigh vacuum. ${ }^{4-6}$ For these Fe(110) films, a strong strain-induced in-plane uniaxial magnetic anisotropy with the easy magnetic axis along the [001] direction has been found. ${ }^{4,7}$ In addition to the cubic magnetocrystalline anisotropy contribution of $K_{1}=6.3 \times 10^{4} \mathrm{~J} / \mathrm{m}^{3}$ a uniaxial magnetic anisotropy of $K_{u}$ $=3.0 \times 10^{4} \mathrm{~J} / \mathrm{m}^{3}$ has been determined.

The $\mathrm{Fe}(110)$ films were patterned into arrays of welldefined magnetic elements by electron-beam lithography (EBL) followed by argon-ion milling. Two different particle shapes have been realized: rectangular and diamond-shaped elements. All elements were $1.5 \mu \mathrm{m}$ long and $0.5 \mu \mathrm{m}$ wide and were separated from adjacent elements in the array by two times their width and length so that magnetostatic interaction between the elements can be neglected. The long axis of the elements was aligned along the easy magnetic axis, i.e., the $\mathrm{Fe}[001]$ direction. In this case the shape, the magnetocrystalline, and the strain-induced uniaxial anisotropy, should lead to a single-domain state in zero field of the ele-

${ }^{\text {a)} E l e c t r o n i c ~ m a i l: ~ r u e d i g e r @ p h y s i k . r w t h-a a c h e n . d e ~}$ ments with the magnetization direction parallel to the long axis.

The magnetization reversal and the magnetic domain states of the microfabricated elements (arrays of $\sim 150000$ identical elements) were investigated by magneto-optic Kerreffect (MOKE) hysteresis loop measurements in a longitudinal geometry and by magnetic-force microscopy (MFM). The CoCr-coated MFM tips were magnetized perpendicular to the surface of the elements, thus mainly the out-of-plane component of the magnetic stray field is detected.

The experimentally determined magnetic domain configurations were compared with micromagnetic simulations performed with the OOMMF $\operatorname{code}^{8}$ software. This program calculates the equilibrium domain states by integrating the Landau-Lifshitz-Gilbert equation for the magnetization dynamics. $^{8}$ The cell size has been chosen as $(5 \mathrm{~nm})^{3}$. The effective field acting on each individual cell is calculated by the derivative of the energy of the system including Zeeman, magnetostatic, exchange, and anisotropy energy terms. The parameters used in the simulation are the saturation magnetization of $M_{S}=1.714 \times 10^{6} \mathrm{~A} / \mathrm{m}$, the exchange stiffness of $A=2.11 \times 10^{4} \mathrm{~J} / \mathrm{m}^{3}$, and the magnetocrystalline as well as stress-induced anisotropy energies of $K_{1}+K_{u}=9.3$ $\times 10^{4} \mathrm{~J} / \mathrm{m}^{3}$. By using a high damping factor of $\alpha=1$ the simulation of quasistatic domain configurations is ensured.

In this letter, we focus on the analysis of the magnetization reversal and the domain states of rectangular and diamond-shaped elements. For this purpose, two different magnetic-field geometries have been investigated. First, with the applied magnetic field along the long axis of the elements (Fe[001] direction), and second, with the in-plane field perpendicular to the easy magnetic axis (Fe[1 10$]$ direction). For the MFM measurements the whole array has been first fully saturated along one of these directions and afterwards the MFM data have been taken in zero-applied field. 


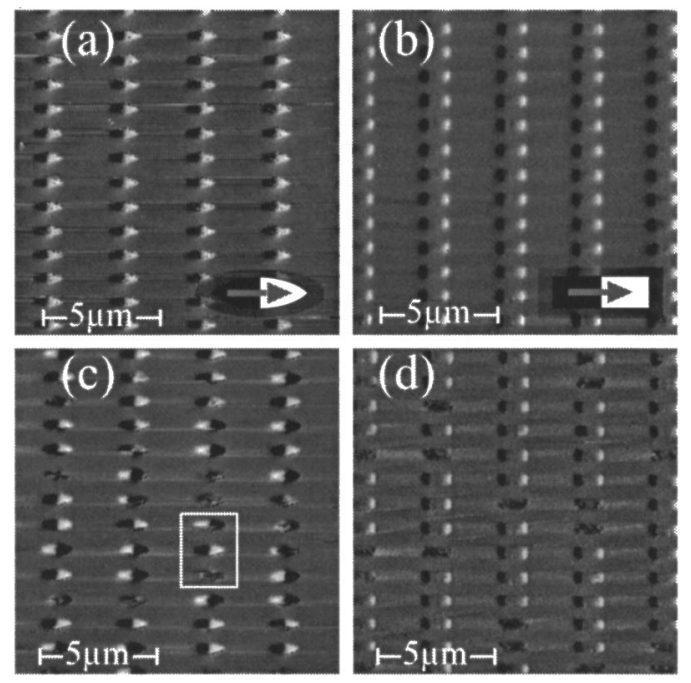

FIG. 1. MFM images at $H=0$ of (a) diamond-shaped and (b) rectangular $\mathrm{Fe}(110)$ elements after magnetic saturation along the long axis. The insets schematically illustrate the dipole-like single-domain states. MFM images at $H=0$ of (c) diamond-shaped and (d) rectangular Fe(110) elements after magnetic saturation perpendicular to the long axis of the elements.

Figures 1(a) and 1(b) show the MFM images of diamond-shaped and rectangular elements at $H=0$, respectively, which were magnetized along the long axis of the elements. Both MFM images present a dipole-like blackand-white contrast indicating a single-domain state, which pictures the stable magnetic domain configuration at $H=0$ during the reversal process along the easy magnetic axis. This observation is confirmed by Kerr hysteresis loop measurements, which show an almost fully remanent magnetization for both types of elements.

In Figs. 2(a) and 2(b), the experimentally determined and simulated hysteresis loops with the field along the easy magnetic axis are shown, respectively. While the diamondshaped elements show an average coercive field of $87 \mathrm{mT}$, the rectangular ones have a coercivity of only $37 \mathrm{mT}$ [Fig. 2(a)]. This trend has been confirmed by the micromagnetic simulations, which give coercive fields of $117 \mathrm{mT}$ for the diamonds and $72 \mathrm{mT}$ for the rectangles [Fig. 2(b)]. The higher coercive field of the diamond-shaped elements is due to the enhanced local shape anisotropy near the needle-like ends, which initially suppresses the nucleation of a reversed domain in the outermost part of the ends. Therefore, the initial magnetization direction of the diamond-shaped elements can be preserved in higher reversed fields. The micromagnetic simulation shows that the reversal process starts in the interior part of the diamond-shaped elements by forming an s-like domain, which propagates to the boundaries by increasing the reversed magnetic field [see the inset of Fig. 2(b)]. Close before reaching saturation along the opposite field direction, the initial magnetization direction still exists near the boundaries and in the outermost parts of the ends.

In the case of the rectangular elements, the large magnetization component perpendicular to the edges of the short sides leads to high magnetic stray fields, so that the nucleation of reversed domains appears at lower reversed fields in contrast to the diamond-shaped elements. The nucleation sites of the reversed domains are located at the corners of the element. After the formation of two vortices, which propa-

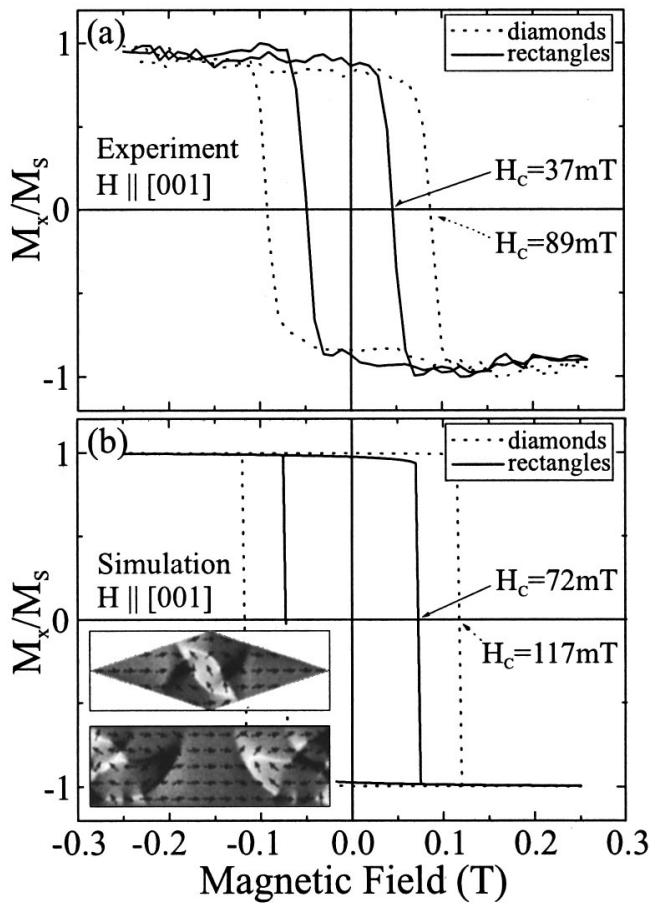

FIG. 2. (a) Experimental and (b) simulated hysteresis loops of rectangular (solid line) and diamond-shaped (dashed line) $\mathrm{Fe}(110)$ elements with the applied field parallel to the long axis of the elements. The insets in (b) show the simulated domain states near the coercive field.

gate to the middle of the rectangular element with increasing reversal field, an annihilation of these vortices occurs when they arrive at the center of the element.

The differences between the experimentally determined coercive fields and the simulated ones [Figs. 2(a) and 2(b)] are due to the nonideal fabrication process of the magnetic elements (crystalline defects in the volume, rough boundaries, smoothed edges, etc.). The simulation of such nonperfectly defined elements (by removing individual cells of the elements) shows a clear reduction of the coercive fields towards the experimental values.

In contrast to the well-defined magnetization direction at $H=0$ after saturation along the easy axis, the same elements show various domain states after saturation perpendicular to the long axis. The marked area of the MFM image in Fig. 1(c) shows three different domain configurations observed in diamond-shaped elements: dipole-like contrast with the magnetization pointing to the left-hand side (top), to the righthand side (middle), and a demagnetized state (bottom). In the case of the rectangular elements, Fig. 1(d) preferably exhibits besides demagnetized elements only dipole-like domain configurations with the magnetization directed to the right-hand side. This is attributed to a small deviation of the applied field from the perfect alignment along the hard magnetic axis during saturation, which gives rise to a small magnetic component parallel to the long axis. The observed demagnetized states in Figs. 1(c) and 1(d) correspond to metastable domain configurations. They represent a snapshot of one phase of the magnetization reversal process. In nonideal structures domain walls can be easily pinned whereby these metastable states can be detected by MFM imaging at $H=0$. Fig. 3 compares various demagnetized states with the corresponding micromagnetic simulations. In Fig. 3(a), the zero-field 

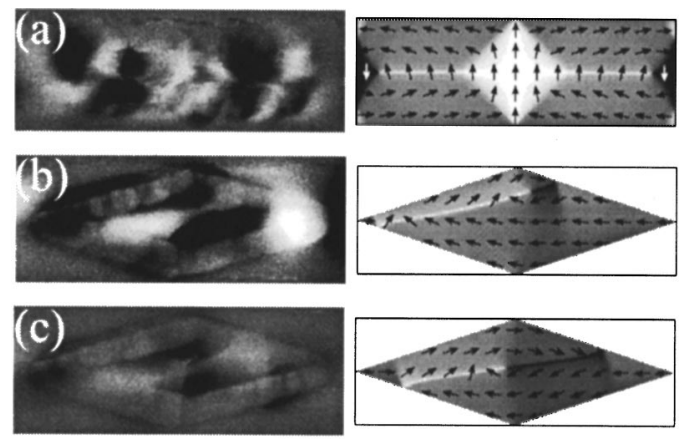

FIG. 3. MFM images and simulated domain states of (a) rectangular and (b) as well as (c) diamond-shaped elements.

MFM image of a demagnetized rectangular element is in close agreement with the simulated domain configuration at an applied reversal field of approximately $60 \mathrm{mT}$. The simulation yields a domain configuration with two counterrotating flux-closure domain states which can also be identified in the MFM image.

The magnification of the demagnetized states of the diamond-shaped elements shows similar deviation between the $H=0$ domain state and the applied-field magnitude for the corresponding simulated domain states. Although the MFM images [Figs. 3(b) and 3(c)] were taken in $H=0$, the experimentally determined domain configurations are comparable with simulations at reversed fields of 35 and $10 \mathrm{mT}$, respectively. In Fig. 3(b), the domain configuration can be interpreted by the presence of a magnetic vortex, which is slightly shifted to the upper part of the element and a pronounced dipole-like structure at the right-hand side, which results in the very bright contrast in the MFM image. The magnetization distribution in Fig. 3(c) is more symmetrical with two smaller dipole-like configurations at the edges with a weaker MFM contrast and a vortex in the center of the element, minimizing the magnetization component perpendicular to the long side of the diamond.

A nonsymmetric magnetization distribution of elements at $H=0$ saturated along the hard axis ( $\mathrm{Fe}[1 \overline{1} 0]$ direction) can also be extracted from the experimental MOKE hysteresis loops. Figure 4(a) shows the hysteresis loops of the diamond-shaped and rectangular element arrays both with a small remanent magnetization at $H=0$. Figure 4(b) exhibits the corresponding micromagneticsimulation of the hard-axis hysteresis loops. In agreement with the simulations, the experimentally determined nucleation fields of the diamondshaped elements array are higher (experimental: $H_{N}$ $\approx 180 \mathrm{mT}$; simulation: $H_{N}=295 \mathrm{mT}$ ) than those of the rectangular elements (experimental: $H_{N} \approx 110 \mathrm{mT}$; simulation: $H_{N}=210 \mathrm{mT}$ ). Due to the enhanced local shape anisotropy of the diamond-shaped elements near the outermost part of the ends, the reversal process starts at higher reversal fields because of the more favorable magnetization direction parallel to the long axis [see the inset of Fig. 4(b)].

We have investigated the magnetization reversal process and the domain states of rectangular and diamond-shaped
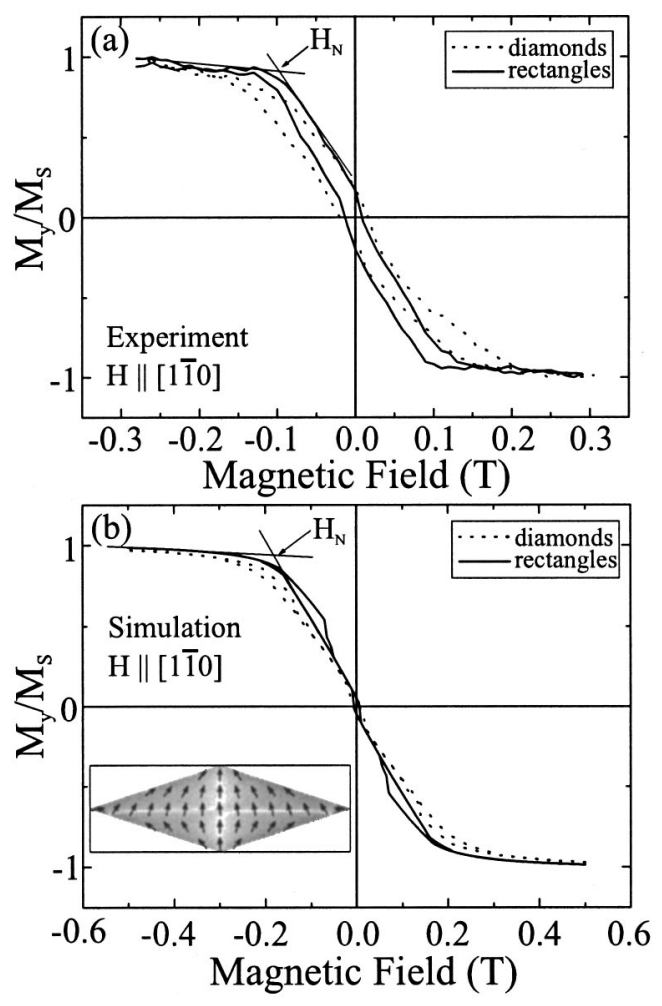

FIG. 4. (a) Experimental and (b) simulated hysteresis loops of rectangular (solid line) and diamond-shaped (dashed line) elements with the field perpendicular to the long axis of the elements. The inset shows the simulated domain state of the diamond-shaped element near the nucleation field.

epitaxial $\mathrm{Fe}(110)$ elements. Independent of the shape of the elements the remanent state after magnetic saturation along the long axis of the elements is a single-domain state. In contrast, after magnetic saturation perpendicular to the long axis, various domain states have been observed. These fluxclosure domain states are in close agreement with micromagnetic simulations. The shape of the elements has a strong influence on the magnetization reversal process, i.e., on the coercive and nucleation fields. In the future, we will correlate the observed domain states with the tunneling magnetoresistance characteristics of $\mathrm{Fe} / \mathrm{AlO}_{x} / \mathrm{Fe}$ junctions.

This work was supported by the EC TMR Program "Submicron Magnetic Structures and Magneto-Transport Devices" (SUBMAGDEV). The work at NYU was supported by DAPRA-ONR, Grant No. N00014-96-1-1207.

${ }^{1}$ W. J. Gallagher, S. S. P. Parkin, Yu Lu, X. P. Bian, A. Marley, K. P. Roche, R. A. Altman, S. A. Rishton, C. Jahnes, T. M. Shaw, and Gang Xiao, J. Appl. Phys. 81, 3741 (1997).

${ }^{2}$ S. S. P. Parkin et al., J. Appl. Phys. 85, 5828 (1999).

${ }^{3}$ T. Schrefl, J. Fidler, K. J. Kirk, and J. N. Chapman, J. Magn. Magn. Mater. 175, 193 (1997).

${ }^{4}$ B. M. Clemens, R. Osgood, A. P. Payne, B. M. Lairson, S. Brennan, R. L. White, and W. D. Nix, J. Magn. Magn. Mater. 121, 37 (1993).

${ }^{5}$ U. Rüdiger, J. Yu, S. Zhang, A. D. Kent, and S. S. P. Parkin, Phys. Rev. Lett. 80, 5639 (1998).

${ }^{6}$ U. May, R. Carlarco, J. O. Hauch, H. Kittur, M. Fonine, U. Rüdiger, and G. Guntherholdt, Surf. Sci. 489, 144 (2001).

${ }^{7}$ J. Yu, U. Rüdiger, A. D. Kent, L. Thomas, and S. S. P. Parkin, Phys. Rev. B 60, 7352 (1999).

${ }^{8}$ http://math.nist.gov/oommf/ 